\title{
DYNAMICAL MODELS FOR CONTROL OF CAVITY OSCILLATIONS
}

\author{
Clarence W. Rowley \\ Tim Colonius \\ Richard M. Murray \\ Division of Engineering and Applied Science \\ California Institute of Technology \\ Pasadena, CA 91125
}

\section{ABstract}

We investigate nonlinear dynamical models for selfsustained oscillations in the flow past a rectangular cavity. The models are based on the method of Proper Orthogonal Decomposition (POD) and Galerkin projection, and we introduce an inner product and formulation of the equations of motion which enables one to use vector-valued POD modes for compressible flows. We obtain models between 3 and 20 states, which accurately describe both the shorttime and long-time dynamics. This is a substantial improvement over previous models based on scalarvalued POD modes, which capture the dynamics for short time, but deviate for long time.

\section{INTRODUCTION}

Self-sustained oscillations over open cavities have been studied extensively since the $1950 \mathrm{~s},{ }^{6,8,10,13,17}$ but recently there has been renewed interest in attenuating these oscillations by means of active control. Many of the recent efforts at controlling cavity oscillations have involved open-loop control $^{16}$ (no feedback), or phase-locked loops, designed to cancel flow disturbances at a particular frequency. ${ }^{2,3,9,14,18}$

None of the control strategies attempted so far have used an explicit dynamical model for control design, or analysis of performance or robustness. In many cases, even a simple low-dimensional model can capture important features of a system, and both indicate effective control strategies, and reveal limitations of active control. The goal of the present work is to formulate and evaluate dynamical models, suitable for bifurcation analysis, that may ultimately be used for control analysis and synthesis.

\subsection{Previous linear models}

Cavity oscillations are thought to arise from a naturally occurring feedback loop: small flow distur-

Copyright (C) 2001 by the authors. Published by the American Institute of Aeronautics and Astronautics, Inc. with permission. bances are amplified by the free shear layer spanning the cavity, scattered into acoustic waves at the cavity trailing edge, propagate upstream, and excite further disturbances in the shear layer. The first description of this feedback process is credited to Rossiter, ${ }^{10}$ who gave a semi-empirical formula for the frequencies of oscillation:

$$
\mathrm{St}_{n}=\frac{n-\gamma}{M+1 / \kappa}, \quad n=1,2,3, \ldots,
$$

where $\mathrm{St}_{n}=f_{n} L / U$ is the Strouhal number corresponding to frequency $f_{n}, L$ is the cavity length, $U$ is the freestream velocity, $M$ is the freestream Mach number, and $\kappa$ and $\gamma$ are empirical constants, related to the phase speed of shear layer disturbances, and the phase shift of the acoustic scattering process, respectively.

Many improvements to Rossiter's original model have been developed, ${ }^{1,6,17}$ but most have been linear models, which can predict frequencies, but not amplitudes, of oscillations. This is a fundamental limitation of linear models, since the loop gain (amplification once around the feedback loop) is independent of amplitude. To capture saturation effects, and predict the correct behavior of finite-amplitude oscillations, a nonlinear model is necessary.

\subsection{Nonlinear models}

A nonlinear tool for predicting amplitudes of cavity oscillations was recently developed by Cain. ${ }^{1}$ It predicts the final amplitude of oscillations, but it does not predict the dynamics - the time evolution of flow disturbances. To perform any analysis of potential controllers for cavity oscillations, one first needs a dynamical model. The focus of this paper is developing such dynamical models for cavity oscillations.

In a previous paper, ${ }^{11}$ we developed dynamical models using the method of Proper Orthogonal Decomposition (POD) and Galerkin projection. This POD/Galerkin method, discussed in detail in section 2, is a standard method for obtaining lowdimensional models, and has been applied extensively to various incompressible flows. ${ }^{7,15}$ In our 
previous paper, we applied these techniques to the compressible cavity flow, but the resulting models had serious limitations: they were accurate for short times ( 2 or 3 periods of oscillation), but deviated significantly for long time. Here, we propose a modified procedure, using vector-valued POD modes instead of scalar valued modes used earlier.

\subsection{Scalar versus vector POD modes}

In incompressible flows, the standard POD/Galerkin method uses vector-valued POD modes for the velocity. The continuity equation requires that the velocity be divergence free, and thus each POD mode must also be divergence free. There is no need to use POD modes for the pressure, as the equations may be rewritten (using the same divergence-free condition) so that the pressure term appears only on the boundaries.

In compressible flow, the situation is different: the velocity is no longer divergence free, and the pressure cannot be eliminated. The continuity equation becomes a separate dynamical equation which must be solved to determine a thermodynamic variable (e.g., density, pressure, temperature, enthalpy). In addition, the energy equation must also be solved, although for isentropic flow these two are redundant.

To overcome this difficulty, in our previous work we used scalar-valued POD modes - separate POD modes for each flow quantity: the velocities $u, v$, and the enthalpy $h$. This leads to separate (but coupled) evolution equations for each flow variable.

In this work, we describe a method for using vector-valued $\mathrm{POD}$ modes, where a single set of $\mathrm{POD}$ modes describes all the flow variables. It is not immediately obvious how to apply this, since the method depends on the structure of an inner product, and it is not obvious how to define such a structure, since one cannot add, say, a velocity and a pressure. This procedure is the core of the paper.

\subsection{Outline}

In section 2, we develop the POD/Galerkin method for an arbitrary inner product. We discuss application of the method to compressible flows in section 3 , defining an inner product, and writing an approximate set of governing equations, valid for cold subsonic flows.

In section 4, we use data from some of our previous Direct Numerical Simulations ${ }^{4}$ (DNS) to compute POD modes, solve Galerkin models, and compare with the full DNS results. We compare the results of the models using vector-valued POD modes to our previous models using scalar-valued POD modes, and also discuss the effect of computing POD modes from snapshots taken while the flow is developing.

\section{Proper Orthogonal Decomposition}

To apply the POD/Galerkin to compressible flow, with vector-valued POD modes, we first need to derive the $\mathrm{POD}$ in a more general setting, for an arbitrary inner product space.

\subsection{POD for arbitrary inner products}

Let $H$ be a Hilbert space, with inner product $\langle\cdot, \cdot\rangle$. The goal is, given an ensemble of data $u(t) \in H$, find an "optimal" subspace $S$ of fixed (finite) dimension, such that the error $E\left(\left\|u-P_{S} u\right\|\right)$ is minimized. Here, $\|\cdot\|$ is the induced norm on $H, P_{S}$ is the projection onto the subspace, and $E(\cdot)$ denotes an average over $t$. This average could be thought of as a time average, or as an ensemble average over many realizations of data, parameterized by $t$. All we will assume is that this averaging procedure is linear and continuous, and thus commutes with the inner product.

Let $n=\operatorname{dim} S$, and let $\left\{\varphi_{j} \in H \mid j=1, \ldots, n\right\}$ be an orthonormal basis for $S$. Then

$$
P_{S} u(t)=\sum_{j=1}^{n}\left\langle u(t), \varphi_{j}\right\rangle \varphi_{j}
$$

and it is simple to show that minimizing the error in the projection, $E\left(\left\|u-P_{S} u\right\|\right)$, over $S$, is equivalent to maximizing the "energy" captured, $\sum_{j=1}^{n} E\left(\left|\left\langle u, \varphi_{j}\right\rangle\right|^{2}\right)$, over $\varphi_{j}$. We now have a constrained variational problem: find orthogonal functions $\varphi_{j}$ which maximize this sum, subject to the constraint $\left\|\varphi_{j}\right\|^{2}=1$. These functions $\varphi_{j}$ are extremals of the functional

$$
J[\varphi]=E\left(|\langle u, \varphi\rangle|^{2}\right)-\lambda\left(\|\varphi\|^{2}-1\right) .
$$

(At this point, it is not obvious that the extremals of $J$ will be orthogonal, but we will see shortly that they are.) Now, an important definition: for $\varphi, \psi \in$ $H$, we denote by $\varphi \otimes \psi: H \rightarrow H$ the linear operator defined by

$$
(\varphi \otimes \psi) \chi=\langle\chi, \psi\rangle \varphi
$$

(Note that $\varphi \otimes \psi$ depends on the inner product.) Then, we may rewrite (3) as

$$
J[\varphi]=\langle R \varphi, \varphi\rangle-\lambda\left(\|\varphi\|^{2}-1\right)
$$


where

$$
R=E(u \otimes u) .
$$

It is simple to show that $R$ is self-adjoint (that is, $\langle R \varphi, \psi\rangle=\langle\varphi, R \psi\rangle$ for all $\varphi, \psi \in H)$. Solving this constrained variational problem leads to the result

$$
R \varphi=\lambda \varphi,
$$

so each optimal basis function $\varphi$ must be an eigenfunction of $R$. We call these eigenfunctions $P O D$ modes. Note that since $R$ is self-adjoint, its eigenvalues are real, and its eigenfunctions may be chosen to be orthonormal.

Now, by taking an inner product with $\varphi$, we see from (7) that

$$
\lambda=E\left(|\langle u, \varphi\rangle|^{2}\right) .
$$

Thus, $R$ is positive semi-definite $(\lambda \geq 0)$, and the functions $\varphi_{j}$ which maximize our original functional $\sum_{j=1}^{n} E\left(\left|\left\langle u, \varphi_{j}\right\rangle\right|^{2}\right)$ are the eigenfunctions corresponding to the largest $n$ eigenvalues of $R$. Furthermore, from (8) we see that the eigenvalues $\lambda_{j}$ give the average "energy" captured by POD mode $\varphi_{j}$, where the energy is in the sense of the induced norm.

\subsection{Computation: method of snapshots}

Often, our data is specified as a collection of snapshots $u_{j} \in H$ where $j=1, \ldots, m$. In this case, $E(\cdot)$ is defined as a weighted average over the snapshots:

$$
E(f(u))=\sum_{j=1}^{m} \beta_{j} f\left(u_{j}\right)
$$

where the weights $\beta_{j}>0$ satisfy $\sum_{j} \beta_{j}=1$. Typically, $\beta_{j}=1 / m$, for equal weighting.

It is easily shown that the POD modes $\varphi$ are linear combinations of snapshots, so we may write

$$
\varphi=\sum_{k=1}^{m} c_{k} u_{k}
$$

for some coefficients $c_{k} \in \mathbb{R}$. Inserting this expansion into the original eigenvalue problem (7), we obtain a finite-dimensional eigenvalue problem

$$
U c=\lambda c,
$$

where $c=\left(c_{1}, \ldots, c_{m}\right)$ and $U$ is an $m \times m$ matrix with

$$
U_{i j}=\beta_{i}\left\langle u_{j}, u_{i}\right\rangle
$$

Note that $U$ is self-adjoint under the inner product $\langle a, b\rangle_{\beta}=\sum_{j} a_{j} b_{j} / \beta_{j}$, so its eigenvectors $c$ are orthogonal under the same inner product.

Typically the functions $u_{j}$ are discretized in space e.g., $u_{j} \in \mathbb{R}^{n}$, where $n$, the number of gridpoints, is usually large. In this case, the original eigenvalue problem (7) has dimension $n$, while the method of snapshots (10) has dimension $m$. The method of snapshots is therefore more efficient whenever the number of snapshots is smaller than the number of gridpoints.

\subsection{Galerkin projection}

Assume our system is governed by a partial differential equation (PDE) of the form

$$
\dot{u}=D_{\mu}(u),
$$

where $u(t) \in H$, and $D_{\mu}$ is a nonlinear differential operator that depends on some parameters $\mu$. In our case, (12) will be the Navier-Stokes equations or the Euler equations, $u(t)$ will be a vector-valued function of space, containing the velocities and a thermodynamic variable (either $h$, the enthalpy, or $a$, the local sound speed), and $\mu$ will contain the parameters we are interested in (e.g., $\mu=(M, L / \theta, \mathrm{Re}))$.

We may compute approximate solutions to (12) by projecting the equations onto a finite-dimensional subspace (e.g., the optimal subspace determined in section 2.1), and solving a simpler set of ordinary differential equations (ODEs) on this subspace. To do this, we expand $u(t)$ in some predetermined basis functions $\varphi_{j}$ (typically POD modes):

$$
u(t)=\sum_{j=1}^{n} a_{j}(t) \varphi_{j} .
$$

Inserting this expansion into (12) and taking an inner product with $\varphi_{k}$ yields

$$
\dot{a}_{k}=\left\langle D_{\mu}(u), \varphi_{k}\right\rangle, \quad k=1, \ldots, n .
$$

Since $u$ is written in terms of the time coefficients by (13), this gives a set of ODEs for $a_{k}$.

Finally, note that for systems with symmetry (e.g., cylindrical jets, homogeneous turbulent flows) it is important to take symmetries into consideration, and that proper treatment of continuous symmetry has been shown to lead to significant computational savings. ${ }^{12}$ See Sirovich ${ }^{15}$ or Holmes et al. $^{7}$ for more information about the POD/Galerkin method.

3 


\section{ApplicAtion to COMPRESSIBLE FLOW}

\subsection{Inner products for compressible flow}

Here we introduce an inner product useful for compressible flow problems. If the flow variables are $q=(u, v, a)$, where $u$ and $v$ are the velocities in 2D and $a$ is the local sound speed, then we define the inner product

$$
\left\langle q_{1}, q_{2}\right\rangle_{\alpha}=\int_{\Omega}\left(u_{1} u_{2}+v_{1} v_{2}+\frac{2 \alpha}{\gamma(\gamma-1)} a_{1} a_{2}\right) d x
$$

where $\alpha$ is a parameter, and $\gamma$ is the ratio of specific heats. If $\alpha=\gamma$ then the induced norm gives $\|q\|^{2}=$ $2 h_{0}$, or twice the total enthalpy of the flow. If $\alpha=1$, then the induced norm gives twice the total energy of the flow.

In this paper, we take $\alpha=\gamma$, and $\Omega$ to be the subset of the computational domain with $-D \leq x \leq$ $L+D$, and $-D \leq y \leq 2 D$, where $D$ is the cavity depth, $L$ is the cavity length, and the origin is at the cavity leading edge.

\subsection{Equations of motion}

In our previous work, ${ }^{11}$ we proposed the following approximate equations of motion, for compressible flow:

$$
\begin{aligned}
& u_{t}+u u_{x}+v u_{y}+h_{x}=\nu\left(u_{x x}+u_{y y}\right) \\
& v_{t}+u v_{x}+v v_{y}+h_{y}=\nu\left(v_{x x}+v_{y y}\right) \\
& h_{t}+u h_{x}+v h_{y}+(\gamma-1) h\left(u_{x}+v_{y}\right)=0 .
\end{aligned}
$$

Here, $u$ and $v$ are velocities, $h$ is the enthalpy, $\gamma$ is the ratio of specific heats ( 1.4 for air), and $\nu$ is the kinematic viscosity, assumed constant. In these equations, we have neglected viscous dissipation and heat transfer, but have included viscous diffusion (assuming the density is constant only in the viscous term). These equations are therefore valid for low Mach numbers, where temperature gradients are not too great.

To use the inner product described above, we transform to the variables $(u, v, a)$, where $a$ is the local sound speed. Using $a^{2}=(\gamma-1) h$, equations (15) become

$$
\begin{aligned}
& u_{t}+u u_{x}+v u_{y}+\frac{2}{\gamma-1} a a_{x}=\nu\left(u_{x x}+u_{y y}\right) \\
& v_{t}+u v_{x}+v v_{y}+\frac{2}{\gamma-1} a a_{y}=\nu\left(v_{x x}+v_{y y}\right) \\
& a_{t}+u a_{x}+v a_{y}+\frac{\gamma-1}{2} a\left(u_{x}+v_{y}\right)=0 .
\end{aligned}
$$

We may also introduce parameters into the equations by rescaling the various flow variables, as in
Rowley et al. ${ }^{11}$ If we nondimensionalize velocities by the freestream velocity $U$, the local sound speed by the ambient sound speed $a_{\infty}$, time by $L / U, x$ by $L$, and $y$ by $\theta$, the momentum thickness at the cavity leading edge, we obtain

$$
\begin{aligned}
& u_{t}+u u_{x}+\frac{L}{\theta} v u_{y}+\frac{1}{M^{2}} \frac{2}{\gamma-1} a a_{x}=\frac{1}{\operatorname{Re}_{\theta}}\left(\frac{\theta}{L} u_{x x}+\frac{L}{\theta} u_{y y}\right) \\
& v_{t}+u v_{x}+\frac{L}{\theta} v v_{y}+\frac{L}{\theta} \frac{1}{M^{2}} \frac{2}{\gamma-1} a a_{y}=\frac{1}{\operatorname{Re}_{\theta}}\left(\frac{\theta}{L} v_{x x}+\frac{L}{\theta} v_{y y}\right) \\
& a_{t}+u a_{x}+\frac{L}{\theta} v a_{y}+\frac{\gamma-1}{2} a\left(u_{x}+\frac{L}{\theta} v_{y}\right)=0
\end{aligned}
$$

where $\operatorname{Re}_{\theta}=\theta U / \nu$. These are the equations of motion we use for Galerkin projection.

\section{Results}

The results are based on a large dataset of Direct Numerical Simulations (DNS) we have performed. The fully compressible 2D Navier-Stokes equations are solved using 6th-order compact finite differences and 4th-order Runge-Kutta time advancement. Typical grids for the 2D simulations have roughly 500,000 gridpoints, and the code has been validated by a careful study of grid resolution and boundary placement. ${ }^{4,5}$

All results here use data from a run with $L / D=2$, $M=0.6, L / \theta=58.4, \operatorname{Re}_{\theta}=68.5$.

\subsection{POD modes}

We compute two different sets of POD modes, using the method of snapshots discussed in section 2.2. For the first set, we take snapshots while the flow is developing, using 201 snapshots between $t=0$ and $t=38.7$. For the second set, we take snapshots after the flow has settled down into oscillations, using 51 snapshots between $t=67.8$ and $t=77.5$. All times are nondimensionalized by $L / U$.

Figure 1 shows the eigenvalues of the POD modes for the two cases. As we expect, the energy decay is much slower when we take snapshots from the developing region, as more modes are necessary to capture the more complicated transient flow structures.

Also note that, especially for the fully-developed POD modes, the eigenvalues occur in pairs. This occurs whenever one has oscillations at a single frequency, and is made more clear by looking at the POD modes themselves.

Figure 2 shows the first six POD modes for the fully-developed case. Because the modes are vector valued, we can compute any flow quantity from them, and here we plot the vorticity and dilatation, which approximately separates convecting disturbances from acoustic waves. 


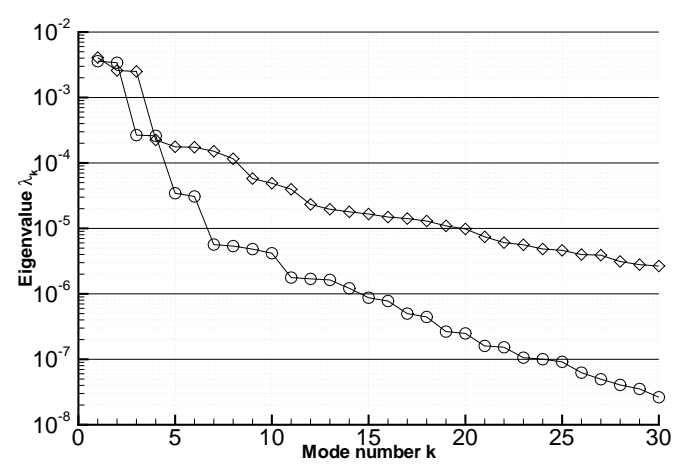

Figure 1: Eigenvalues of POD modes, for snapshots taken in transient regime $(\diamond)$, and after flow has fully developed (०).

Now it is more clear why the POD modes occur in pairs: the oscillations are approximately sinusoidal, at a few resonant frequencies, so the POD modes occur in pairs as $90^{\circ}$ phase-shifted versions of each other, much like sine and cosine. The frequency of the first two POD modes corresponds to Rossiter mode $2(n=2$ in equation (1)), and it is clear there are two wavelengths of oscillations in the shear layer, consistent with Rossiter's description.

Higher modes contain smaller and smaller scales, at higher temporal frequencies, with correspondingly smaller acoustic wavelengths.

The POD modes from the transient dataset look similar, but do not occur in such neat pairs, as they contain short-lived flow structures which arise as the flow develops.

\subsection{Galerkin models}

Figure 3 shows the results of a simulation with scalar-valued POD modes, presented in our previous paper. ${ }^{11}$ Note that here, a "4 mode" model requires 4 modes for each flow variable $(u, v, h)$, resulting in 12 modes altogether, and 12 ODEs to be solved. The initial condition is obtained by projecting a snapshot from the DNS (the first snapshot used for the POD computation) onto the POD modes. We compare the evolution of $u$-velocity mode 1 with the projection of the DNS onto this same mode.

All of the scalar-valued models are accurate for short time, but deviate significantly for longer time. Furthermore, taking more modes does not always help: for long times, the most accurate model is the 2-mode model! The 10-mode model actually blows up, and the 20-mode model looks qualitatively very

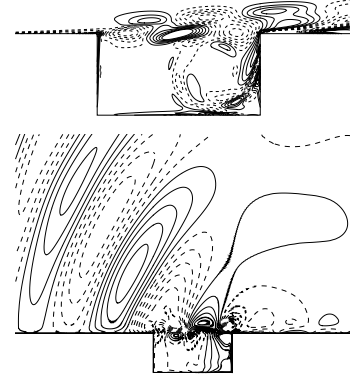

(a) Mode $1(47.15 \%)$
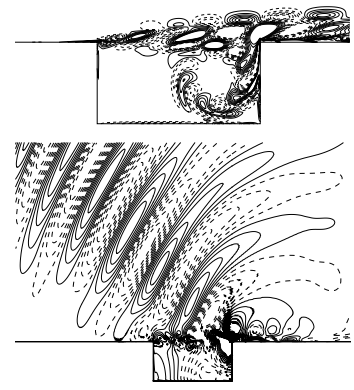

(c) Mode $3(3.50 \%)$

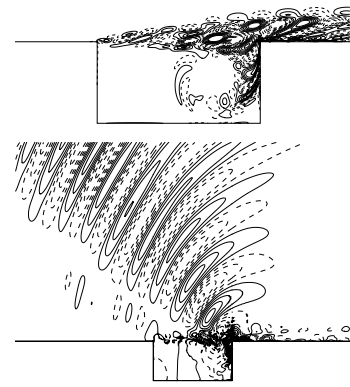

(e) Mode $5(0.45 \%)$

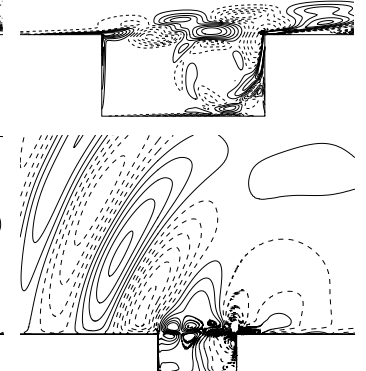

(b) Mode $2(44.67 \%)$

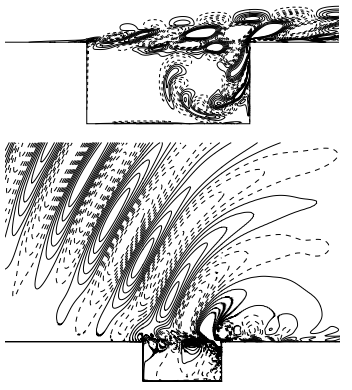

(d) Mode $4(3.42 \%)$

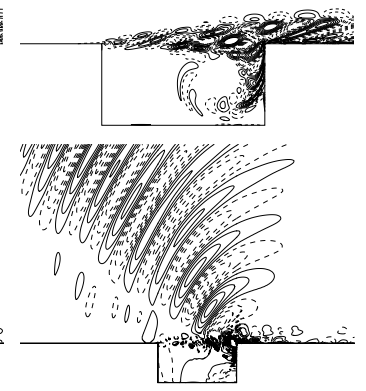

(f) Mode $6(0.40 \%)$
Figure 2: Vorticity (top) and dilatation (bottom) for vector-valued POD modes (snapshots from fully developed flow), and percent energy captured. Negative contours are dashed. 

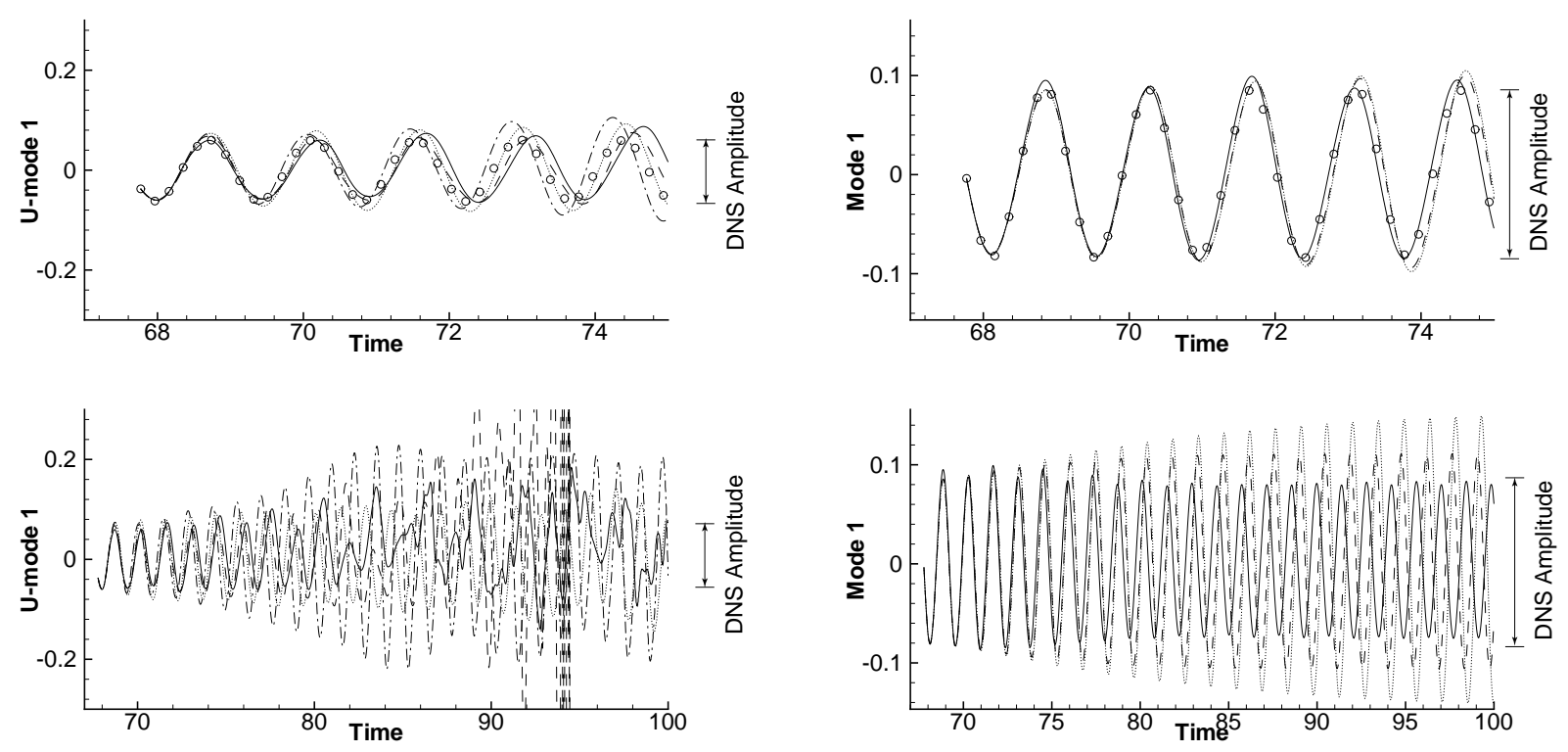

Figure 3: Scalar POD modes: coefficient of $u$ velocity mode 1 , for projection of DNS (o), and POD/Galerkin models with 2 modes (…...), 4 modes (-- - ), 10 modes (----), and 20 modes (-). The two plots are from the same simulation, for different time intervals.

different for long time, with a complicated, chaoticlooking waveform.

Figure 4 shows the results of the same simulation using vector-valued POD modes. The same snapshots are used as in figure 3, and the same snapshot is projected onto the modes for the initial condition. Also, here since there is only one set of POD modes, the number of ODEs to solve is fewer by a factor of 3 compared to the scalar-valued method.

The simulations are all very accurate for short time, and also qualitatively accurate for long time. The 4-mode case drifts to a larger amplitude for long time, and the 6-mode and 11-mode cases are closer. This is in sharp contrast to the scalar mode case, where taking more modes made the dynamics worse for long time. We explain possible reasons for this in section 5 .

We also note here that using the POD modes from the developing region gave worse results than using the fully-developed POD modes: more modes are required for qualitatively accurate results, and furthermore, taking more modes tends to make the models unstable, as with scalar modes shown in figure 3. This is surprising, as one might think that the

Figure 4: Vector POD modes: coefficient of mode 1, for projection of DNS (०), and POD/Galerkin models with 4 modes (…..), 6 modes (----), and 11 modes (-). The two plots are from the same simulation, for different time intervals.

developing POD modes might make Galerkin projections more stable: since they contain more information about the transients, they might be better able to correct if the solution started to stray from its steady-state conditions. It is likely that many more modes are necessary, however, given the much slower energy decay shown in figure 1 . The highest number of modes we tried in our simulations with transient POD modes was 15, and though the first 15 transient POD modes capture $98.57 \%$ of the energy in the transient dataset, they presumably capture much less energy for the fully developed oscillations.

\subsection{Transient regime}

We now compare the behavior of the Galerkin models, when they are started at an initial condition far from their steady state. Figures 5 and 6 show the behavior of the Galerkin models using fullydeveloped POD modes, and transient POD modes, respectively. The initial condition is the same initial condition that was used in the DNS: a Blasius boundary layer spanning the cavity.

The fully-developed POD modes, shown in Figure 5, are not quantitatively accurate for short times, but they do surprisingly well for long times. All 

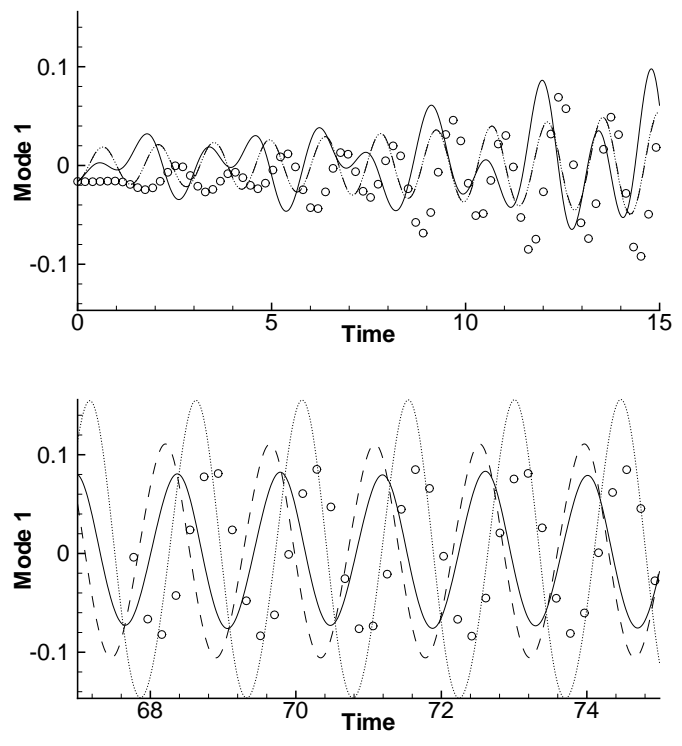

Figure 5: Snapshots from fully-developed region: coefficient of (vector-valued) mode 1 , starting with initial condition at $t=0$. The bottom plot is from the same simulation, at a later time. Symbols are as in Figure 4. Note that in the top plot, the dashed and dotted lines are practically identical.
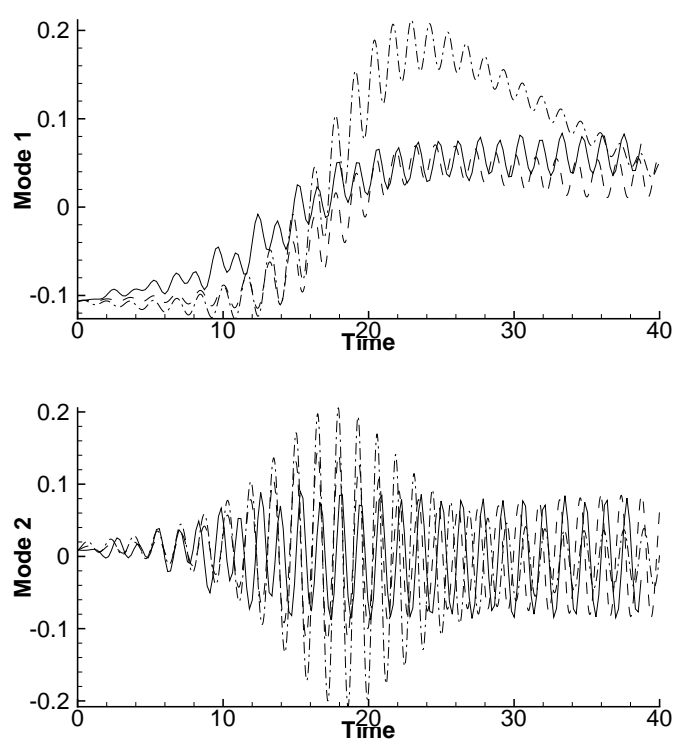

Figure 6: Snapshots from developing region: Projection of DNS (-), and POD/Galerkin models with 3 modes $(---)$, and 6 modes (---). Here, different POD modes were used for the projection, taking snapshots from the developing region. three simulations $(4,6$, and 11 modes) capture the right frequency and amplitude for long times, and the qualitative behavior is correct: oscillations growing in amplitude, eventually saturating.

The transient POD modes do much better, as shown in Figure 6. For the transient POD modes, both modes 1 and 2 are shown. The 3-mode simulation overshoots the amplitude, but eventually settles back to the correct steady-state amplitude (after about $t=110$, well past what is shown in the figure). The 6-mode simulation is quite accurate for all times.

\subsection{Parameter variation}

Here we briefly discuss the effect of varying the Mach number $M$ in the Galerkin models. In particular, we investigate how the frequency of oscillation varies as the Mach number is changed.

We linearize the Galerkin equations obtained by projecting (17) onto the fully-developed POD modes (retaining the parameters in the projected equations). The eigenvalues of the linearized system are shown in Figure 7, for Galerkin models with 4, 6, 11, and 20 POD modes. The 4- and 6-mode models have one pair of unstable (right-half-plane) eigenvalues, at the frequency of Rossiter mode 2. The 10and 20-mode models have an additional pair of unstable eigenvalues, at the lower frequency of Rossiter mode 1. This indicates that Rossiter mode 1 is still present in the dataset, but at a much smaller energy than Rossiter mode 2.

As the Mach number is varied, the eigenvalues move, as shown in figure 7 . When the Mach number is decreased, the eigenvalues become more stable, which is consistent with observations from the simulations.

Figure 8 shows how the frequencies (imaginary parts of the unstable eigenvalues) change as the Mach number is varied. Also plotted are the frequencies measured from the DNS at $M=0.6$, and the curves of the first two frequencies predicted by Rossiter's formula (1), using Rossiter's original values $\gamma=0.25,1 / \kappa=1.75$. The frequencies closely match the frequency measured in the DNS, and capture the same trend with Mach number predicted by Rossiter's model. Note that we do not expect our Galerkin model to be valid very far from the parameter range where the snapshots were taken, since for different parameter values, the flow structures will presumably be different. 


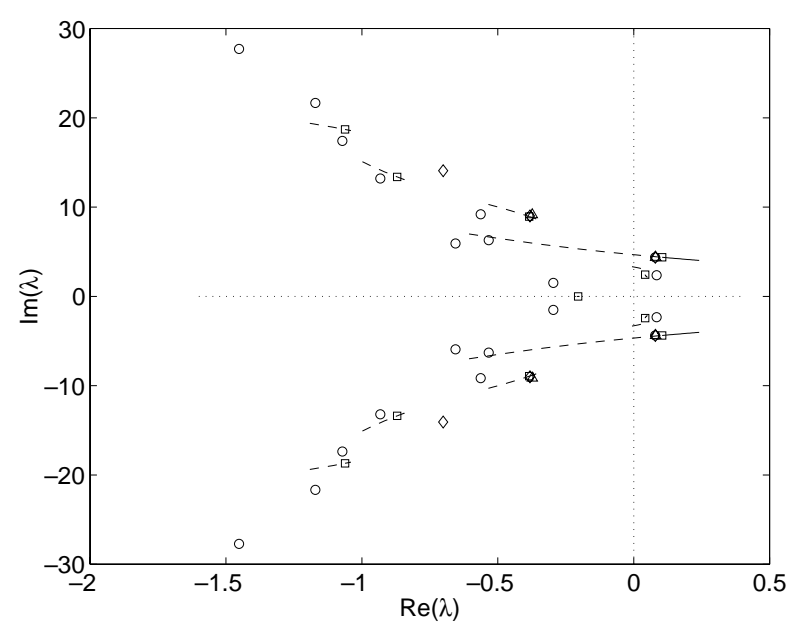

Figure 7: Eigenvalues of 4-mode $(\triangle), 6$-mode $(\diamond), 11$ mode ( $\square$ ), and 20-mode (०) linearized Galerkin models, at the nominal value $M=0.6$; and of the 11-mode model with $0.3<M<0.6$ (----), and $0.6<M<0.9$ (—).

\section{Conclusions}

We have presented dynamical models for selfsustained oscillations in the compressible flow past a rectangular cavity, based on the POD/Galerkin method. We have introduced an inner product and a formulation of the equations of motion which enables one to use vector-valued POD modes for compressible flow. The vector-valued modes capture the coherent structures in the shear layer that spans the cavity, as well as the corresponding acoustic radiation, and the resulting Galerkin projections capture the cavity dynamics well, for both short and long time. This is a substantial improvement over models obtained from scalar-valued POD modes, which tended to deviate for long time. We have also looked briefly at how the frequencies of oscillation of our models depend on Mach number, and we have found a reasonable agreement with both direct numerical simulations and Rossiter's formula.

A plausible reason why the vector-valued modes do better than scalar modes is the following: certain terms in the equations, namely the dilatation $u_{x}+v_{y}$, are sensitive, in that they involve a delicate cancellation between two large numbers (even though $u_{x}$ and $v_{y}$ are not small, the dilatation $u_{x}+v_{y}$ always remains small). If scalar-valued modes are used, $u$ and $v$ may drift apart slightly after some time, so that they no longer cancel each other, and the resulting error in dilatation can drive the equations

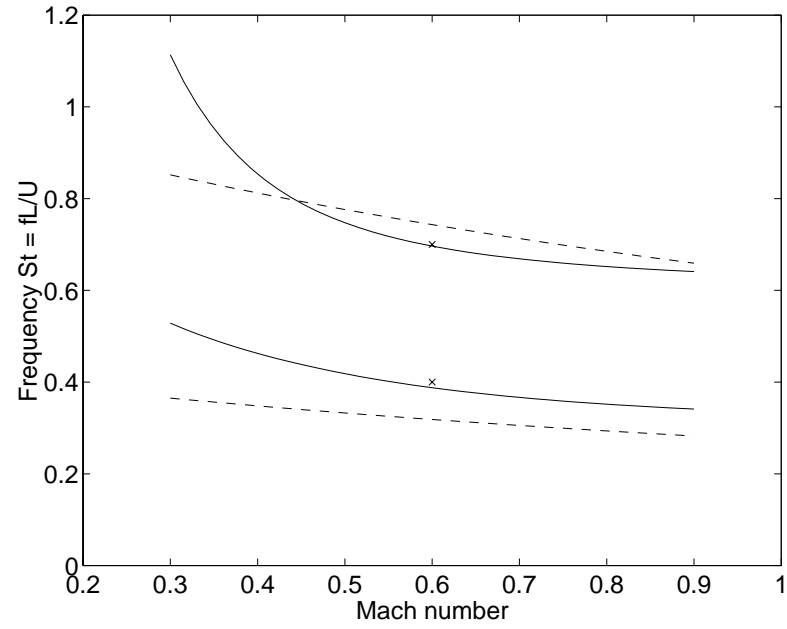

Figure 8: Frequencies predicted by Galerkin model as Mach number is varied: $\operatorname{Im}(\lambda / 2 \pi)$ for unstable eigenvalues $\lambda$ of 11-mode Galerkin model (frequencies measured from DNS $(\times)$; and frequencies predicted by Rossiter equation (----).

in a non-physical way. We have observed this effect directly in Galerkin simulations from scalar-valued POD modes. When vector-valued modes are used, the dilatation is computed much more accurately, as it is effectively computed for each POD mode, and not from a sum of POD modes for different variables which may drift apart.

In order to use these models for control analysis or synthesis, it is of course necessary to introduce the effects of actuation into the models. The presence of an actuator will presumably change the flow structures, so POD modes need to be taken in the actuated flow, perhaps stacking snapshots from different runs. Precisely how to do this remains an open question, and is a topic for future research, but the present results are promising: since the unactuated flow can be accurately modeled by as few as 3 POD modes, it is likely the actuated flow may also be described by a model of very low dimension.

\section{ACKnowledgements}

This work was supported by AFOSR under grants F49620-98-1-0095 and F49620-95-1-0419. The first author gratefully acknowledges a NSF graduate research fellowship.

\section{REFERENCES}

[1] A. B. Cain, W. W. Bower, F. McCotter, and W. W. Romer. Modeling and prediction of 
weapons bay acoustic amplitude and frequency. Technical report, VEDA Inc., 1996.

[2] L. N. Cattafesta III, D. Shukla, S. Garg, and J. A. Ross. Development of an adaptive weapons-bay suppression system. AIAA Paper 99-1901, May 1999.

[3] T. Colonius. An overview of simulation, modeling, and active control of flow/acoustic resonance in open cavities. AIAA Paper 2001-0076, 2001.

[4] T. Colonius, A. J. Basu, and C. W. Rowley. Numerical investigation of the flow past a cavity. AIAA Paper 99-1912, May 1999.

[5] T. Colonius, A. J. Basu, and C. W. Rowley. On self-sustained oscillations in twodimensional compressible flow over rectangular cavities. Journal of Fluid Mechanics, submitted, 1999.

[6] H. H. Heller and D. B. Bliss. The physical mechanism of flow induced pressure fluctuations in cavities and concepts for their suppression. AIAA Paper 75-491, 1975.

[7] P. Holmes, J. L. Lumley, and G. Berkooz. Turbulence, Coherent Structures, Dynamical Systems and Symmetry. Cambridge University Press, 1996.

[8] K. Krishnamurty. Sound Radiation from Surface Cutouts in High Speed Flow. PhD thesis, California Institute of Technology, 1956.

[9] L. Mongeau, H. Kook, and M. A. Franchek. Active control of flow-induced cavity resonance. AIAA/CEAS Paper 98-2349, 1998.

[10] J. E. Rossiter. Wind-tunnel experiments on the flow over rectangular cavities at subsonic and transonic speeds. Aeronautical Research Council Reports and Memoranda, No. 3438, Oct. 1964.

[11] C. W. Rowley, T. Colonius, and R. M. Murray. POD based models of self-sustained oscillations in the flow past an open cavity. AIAA Paper 2000-1969, June 2000.

[12] C. W. Rowley and J. E. Marsden. Reconstruction equations and the Karhunen-Loève expansion for systems with symmetry. Physica D, 142:1-19, 2000.
[13] V. Sarohia. Experimental and Analytical Investigation of Oscillations in Flows Over Cavities. $\mathrm{PhD}$ thesis, California Institute of Technology, 1975.

[14] L. Shaw and S. Northcraft. Closed loop active control for cavity acoustics. AIAA Paper 991902, May 1999.

[15] L. Sirovich. Turbulence and the dynamics of coherent structures, parts I-III. Quarterly of Applied Mathematics, XLV(3):561-590, Oct. 1987.

[16] M. J. Stanek, G. Raman, V. Kibens, J. A. Ross, J. Odedra, and J. W. Peto. Control of cavity resonance through very high frequency forcing. AIAA Paper 2000-1905, June 2000.

[17] C. K. W. Tam and P. J. W. Block. On the tones and pressure oscillations induced by flow over rectangular cavities. Journal of Fluid Mechanics, 89(2):373-399, 1978.

[18] D. R. Williams and D. Fabris. Experiments on controlling multiple acoustic modes in cavities. AIAA Paper 2000-1903, June 2000. 